\title{
Shining light on multi-drug resistant Candida auris: ultraviolet-C disinfection, wavelength sensitivity, and prevention of biofilm formation of an emerging yeast pathogen
}

\author{
Richard Mariita $^{1}$, James Davis ${ }^{1}$, Michelle Lottridge ${ }^{2}$, and Rajul Randive ${ }^{1}$ \\ ${ }^{1}$ Crystal IS Inc., an Asahi Kasei company \\ ${ }^{2}$ Crystal IS Inc., an Asahi Kasei company
}

October 25, 2021

\begin{abstract}
Background: Candida auris is an emerging fungal pathogen of worldwide interest. It is associated with high mortality rates and exhibits increased resistance to antifungals. Ultraviolet-C (UVC) light can be used to disinfect surfaces to mitigate its spread. In this study, we analyzed the performance of different UVC wavelengths against C. auris to determine its wavelength sensitivity and UVC dose requirements and evaluated biofilm prevention dose requirements on most used materials in healthcare settings. Objectives: 1. To investigate UVC disinfection performances and wavelength sensitivity of C. auris; 2 . To evaluate the UVC dose required for prevention of biofilm prevention on stainless steel. Methods: C. auris was grown following standard procedures. The study utilized six different UVC LED arrays with wavelengths between 252 and $280 \mathrm{~nm}$. Arrays were set at similar intensities, to obtain doses of 5-40 mJcm-2 and similar irradiation time. Disinfection performance for each array was determined using log reduction value (LRV) and percentage reduction by comparing the controls against the irradiated treatments. Evaluation of the ability of $267 \mathrm{~nm}$ UVC LEDs to prevent C. auris biofilm formation was investigated using stainless steel, plastic coupons, and poly-cotton fabric. Results: Peak sensitivity to UVC disinfection was between 267 and $270 \mathrm{~nm}$. With $20 \mathrm{mJcm}-2$, the study obtained LRV 3. On steel coupons, $30 \mathrm{mJcm}-2$ was sufficient to prevent biofilm formation, on plastic only $10 \mathrm{mJcm}-2$. A dose of $60 \mathrm{mJcm}-2$ reduced biofilms on poly-cotton fabric significantly. Conclusions: Results revealed that C. auris was most susceptible at 267-270 nm. Additional highlights from the study allow for the design and implementation of disinfection systems.
\end{abstract}

\section{Hosted file}

Candida_auris_MYA-5001_manuscript_.docx available at https://authorea.com/users/442926/ articles/543038-shining-light-on-multi-drug-resistant-candida-auris-ultraviolet-cdisinfection-wavelength-sensitivity-and-prevention-of-biofilm-formation-of-an-emergingyeast-pathogen 